\title{
3D Eddy-Current Imaging of Metal Tubes by Gradient-Based, Controlled Evolution of Level Sets
}

\author{
Juan Felipe P J Abascal, Marc Lambert, Dominique Lesselier, Senior Member, IEEE, and Oliver Dorn
}

\begin{abstract}
Eddy-current non-destructive testing is widely used to detect defects within a metal structure. It is also useful to characterize their location and shape provided that proper maps of variations of impedance which the defects induce are available. Imaging of void defects in the wall of a hollow, nonmagnetic metal tube, is performed herein by controlled evolution of level sets. Such data are variations of impedance collected by a circular probe array close to the inner surface of the tube when a coil source operated at one single frequency is set along its axis at some distance from the array, both receiver and coil source being moved simultaneously. The defect zone is represented in implicit fashion as a zero level set, amenable to topological changes via a nonlinear iterative method that minimizes a least-square cost functional made of the difference between the measured (computer simulated) and model data. The procedure involves the rigorous calculation of the gradient of the variations of impedance, in the case of a multi-static configuration (driver and receiver coils are different), a vector domain integral field formulation being used to that effect. Numerical examples, via a dedicated extension of the generalpurpose CIVA platform, exhibit pros and cons of the approach for inner, outer, and through-wall void defects, with further comparisons to results provided by an independently-developed binary-specialized method.
\end{abstract}

Index Terms - eddy-current non-destructive testing, impedance variations, level-sets, optimization of topology, imaging

\section{INTRODUCTION}

$\mathbf{E}$ DDY current testing of metal tubes is of interest for quality examination of highly conductive material in production lines or for in-service inspection of industrial parts. The interaction between a low-frequency time-harmonic electromagnetic field produced by a source probe and one or more defects induces a perturbation of eddy currents which is seen, most often, from the variation of impedance of a receiver probe nearby. In a recent investigation, it has been shown how one can express this variation of impedance via the reciprocity theorem from a vector domain integral formulation involving a set of Green's dyads, and calculate it by a carefully tailored method of moments [1]. The resulting computer code has been implemented into the CIVA platform (http://www-civa.cea.fr) and can be used to address conductive and/or permeable metal tubes in the time-harmonic eddy current regime for a wide range of multistatic configurations (separated sources and receivers).

Juan Felipe P-J Abascal, Marc Lambert, and Dominique Lesselier are with Département de Recherche en Electromagnétisme, Laboratoire des Signaux et Systèmes UMR8506 (CNRS-Supélec-Univ Paris Sud 11), 91192 Gif-surYvette cedex, France

Oliver Dorn is with Grupo de Modelización y Simulación, Universidad Carlos III de Madrid, Avda de la Universidad, 30, 28911 Leganés, Madrid, Spain
In the present paper, starting from this know-how on eddycurrent models, one is investigating the mapping of a 3D bounded (volumetric) void defect affecting the tube wall, assumed to be comprised in full within a certain Region of Interest (RoI). This is carried out for a non-magnetic metal tube, the assumption of a magnetic behavior adding to the complexity of the numerical analysis without much benefit in the present study aiming first at "proof of concept". The solution methodology lies within the realm of controlled evolution of levels sets or topological optimization as reviewed in detail in [2], well-known text books [3], [4] and an abundant literature (out of our scope here) showing the versatility and efficiency of such approaches.

Level sets have been proposed as a model of the interface of an obstacle easily handling topological changes [5]. Defining a level-set function positive inside the domain enclosed by the obstacle and negative outside (or vice versa), the obstacle interface is but the zero level set. A usual representation of the level set is a signed distance function defined as the Euclidean distance from every point in a search space containing the obstacle to the interface, a positive sign being imposed inside (negative outside). Evolution of the level-set function, and implicitly of the interface, can be shown as being governed via a Hamilton-Jacobi equation, which relates the (pseudo-) time evolution of the level set to its velocity, formally derived, e.g., via shape optimal design [6], as in particular shown in [7]. As an alternative to this Hamiltonian approach, a gradientbased level set method can be developed, e.g., [8]. A number of references deal with like reconstructions [9], [10]. The approach below follows that line of thought.

One has to retrieve the shape and location of the defect(s) the surface boundary of which is implicitly defined by a level set function, which is associated to a contrast-of-conductivity function valued to -1 inside them and 0 outside. The level set is initialized in the RoI as a signed distance function, and is henceforth evolved by a nonlinear iterative gradientbased method, which minimizes a least-squares functional of the data-model misfit. The gradient of the variation of impedance with respect to the level set is derived, which is done in a manner similar to the earlier contribution of [11] (with no mention of level sets at this time). This implies the solution of one forward problem and one adjoint problem with similar mathematical and numerical structure. As for the update, the search direction is of the Polak-Ribière conjugategradient type, the step size being restricted so as the contrast is updated in no more than three of the voxels describing the defect (to avoid large updates); further regularization is imposed by applying a spatial filter onto the gradient direction. 
The corresponding numerical machinery is expected to work whether the defect opens in air (inside or outside the tube) or not, or even traverses it, though, in theoretical terms, the assumption that the zero level set is allowed to touch the boundary of the RoI remains delicate matter.

The paper is organized as follows. In section II the ingredients of the forward problem are proposed, heavily borrowing from [1], with specialization to the specific configuration of our interest. In section III the level-set method is discussed in detail, with most emphasis placed on the calculation of the gradient of the variation of impedance. Since, for comparison, one has also developed a binary-specialized method in harmony with the imaging of defects in a planar non-magnetic metal plate [12], with some know-how from [13], this method is summarized as well. Then, the practical implementation of the level-set evolution is discussed. In section IV, a set of numerical results is proposed for various defect topologies. This is carried out from data simulated using the full vector formulation, the azimuthal component of the secondary sources being the one used in the imaging procedure in most cases, taking advantage of the fact that the primary electric field, in applications of our main concern, is null along both radial and axial directions. Such results are concluded upon in section V.

\section{THE FORWARD PROBLEM}

\section{A. The modeling}

Let us consider a hollow circular cylindrical tube (one introduces circular cylindrical coordinates, the vertical $z$ axis coinciding with the tube axis). Assuming only non-magnetic media (with vacuum permeability $\mu_{0}$ ), one denotes by $\sigma_{0}$ the constant conductivity of the tube wall, air being on both sides. With implied time-dependence $e^{-j \omega t}$, the complex permittivity in the eddy-current regime of our interest reads as $-\frac{\sigma}{j \omega}$. Accordingly, the wave number $k$ in metal (with strictly positive imaginary part) is such that $k^{2}=j \omega \sigma \mu_{0}$. A 3-D bounded (volumetric) defect with finite support domain $V_{f}$ and conductivity $\sigma(\vec{r})$ is assumed in the tube wall (its parameters are allowed to vary as a function of location $\vec{r}$ though only homogeneous voids are dealt with in practice). It is characterized at any $\vec{r}$ by the contrast function

$$
\chi(\vec{r})=\frac{\sigma(\vec{r})-\sigma_{0}}{\sigma_{0}} .
$$

The latter is valued to zero outside $V_{f}$. From eddy-current specialization of the Maxwell equations, by applying the Green's theorem and accounting for the usual boundary conditions at material discontinuities and radiation condition at infinity, one shows that the electric field satisfies the vector domain integral formulation [14]-[16]:

$$
\begin{aligned}
& \vec{E}(\vec{r})=\vec{E}^{i n c}(\vec{r})+ \\
& j \omega \mu_{0} \int_{V_{f}} \overline{\overline{\mathbf{G}}}^{(e e)}\left(\vec{r}, \vec{r}^{\prime}\right) \cdot \sigma_{0} \chi\left(\vec{r}^{\prime}\right) \vec{E}\left(\vec{r}^{\prime}\right) d V^{\prime} .
\end{aligned}
$$

The dyadic Green's function $\overline{\overline{\mathbf{G}}}^{(e e)}$ in the above, $\vec{r}$ and $\vec{r}^{\prime}$ being the observation and defect points, resp., is made of the electric field response to a unit point current source of electric nature (with orientations along the three axes of coordinates). It satisfies the dyadic Helmholtz equation

$$
\nabla \times \nabla \times \overline{\overline{\mathbf{G}}}^{(e e)}\left(\vec{r}, \vec{r}^{\prime}\right)-k^{2} \overline{\overline{\mathbf{G}}}^{(e e)}\left(\vec{r}, \vec{r}^{\prime}\right)=\overline{\overline{\mathbf{I}}} \delta\left(\vec{r}-\vec{r}^{\prime}\right),
$$

$\overline{\overline{\mathbf{I}}}$ as the unit dyad, and the reciprocity relationship

$$
\overline{\overline{\mathbf{G}}}^{(e e)}\left(\vec{r}, \vec{r}^{\prime}\right)=\left[\overline{\overline{\mathbf{G}}}^{(e e)}\left(\vec{r}^{\prime}, \vec{r}\right)\right]^{T},
$$

where superscript $T$ stands for transposition. As is usual, one introduces fictitious current sources, null outside $V_{f}$, such as

$$
\vec{J}(\vec{r})=\sigma_{0} \chi(\vec{r}) \vec{E}(\vec{r}),
$$

the electric field being $\vec{E}=\vec{E}^{i n c}+\vec{E}^{s}, \vec{E}$ the total field, $\vec{E}^{s}$ the anomalous one, $\vec{E}^{i n c}$ the primary one (existing in a flawless tube wall), calculated for axisymmetric source coils (one will limit ourselves to one of those) according to the Dodd and Deeds approach [17]).

Inside the defect volume $V_{f}$ equation (2) becomes

$$
\begin{aligned}
& \vec{J}^{i n c}(\vec{r})=\vec{J}(\vec{r})- \\
& j \omega \mu_{0} \sigma_{0} \chi(\vec{r}) \int_{V_{f}} \overline{\overline{\mathbf{G}}}^{(e e)}\left(\vec{r}, \vec{r}^{\prime}\right) \cdot \vec{J}\left(\vec{r}^{\prime}\right) d V^{\prime},
\end{aligned}
$$

letting $\vec{J}^{i n c}$ be the fictitious current (5) associated to the primary field.

Various set-ups can be chosen for the evaluation of a damaged tube wall. Here, the tube is tested from the inside, an axisymmetric horizontal coil being moved along the $z$ axis (the velocity is low enough to ignore motion-related induction). The driving coil (Tx) is fed by an electric current with given amplitude $I_{1}$. Primary eddy currents induced in the tube wall are modified by the defect. A receiving coil ( $\mathrm{Rx})$ sees it as a variation of voltage, or equivalently of mutual Tx-Rx impedance, which follows from the reciprocity theorem [13], [18]:

$$
Z=-\frac{1}{I_{1} I_{2}} \int_{V_{f}} \vec{E}_{\mathrm{Rx}}^{i n c}\left(\vec{r}^{\prime}\right) \cdot \vec{J}\left(\vec{r}^{\prime}\right) d V^{\prime},
$$

where $\vec{E}_{\mathrm{Rx}}^{\text {inc }}(\vec{r})$ denotes the primary electric field induced within the flawless tube wall by the receiving coil (Rx) fictitiously operated in the transmission mode (with current set to $I_{2}$ ). Let us notice that the latter requires the careful calculation of a Green's dyad having source point inside the tube and observation point inside the wall.

Expression (7) corresponds to a single impedance measurement. Imaging is expected to require several positions of receiver and/or transmitter. Let $Z_{i j}$ be the impedance corresponding to receiver $\operatorname{Rx}_{j}$ and transmitter $\operatorname{Tx}_{i}$, with $j=$ $1, \ldots, L$ and $i=1, \ldots, I$ (a total of $I \times L$ measurements), then

$$
Z_{i j}=-\frac{1}{I_{1} I_{2}} \int_{V_{f}} \vec{E}_{\mathrm{Rx}_{j}}^{i n c}\left(\vec{r}^{\prime}\right) \cdot \vec{J}_{i}\left(\vec{r}^{\prime}\right) d V^{\prime},
$$

where $\vec{J}_{i}$ is the fictitious current corresponding to the $i$ thapplied current $\vec{J}_{i}^{i n c}$. 


\section{B. The discrete solution}

Integral equations (6) and (7) are solved by a Method of Moments using pulse basis functions and discretizing the defect with a regular cylindrical grid of $N$ elements [1]. Current (6) satisfies a linear system of equations

$$
\mathbf{S} \mathbf{J}=\mathbf{J}_{0},
$$

where $\mathbf{J}$ and $\mathbf{J}_{0}$ are $3 N \times I$ matrices, for $I$ applied currents, the elements of which are the basis function coefficients, and $\mathrm{S}$ is a $3 N \times 3 N$ matrix.

The linear system (9) can be solved by a direct method, which is faster than an iterative method for small domains, a single inversion of the system matrix being needed for all transmitter positions. This is the case for the inverse problem at hand since the defect domain is necessarily smaller than the RoI, and since the matrices in (9) can be constrained to the defect domain, $\chi \overline{\overline{\mathbf{G}}}^{(e e)}$ and $\vec{J}$ being zero outside it.

\section{Reduction to the azimuthal component}

Measured data are simulated by the full-vector formulation (6),(8), but, for carrying out the inversion, dimensionality is reduced, the azimuthal-azimuthal block of the dyad being the only one accounted for, as follows. Indeed, for the axisymmetric source coil envisaged, the primary electric field is zero along both radial and axial directions $\left(J_{0 \rho}(\vec{r})=J_{0 z}(\vec{r})=0\right)$. Thus, the linear system (9) simplifies into

$$
\left[\begin{array}{c}
\mathbf{J}_{\rho} \\
\mathbf{J}_{\varphi} \\
\mathbf{J}_{z}
\end{array}\right]=\left[\begin{array}{ccc}
\mathbf{T}_{\rho \rho} & \mathbf{T}_{\rho \varphi} & \mathbf{T}_{\rho z} \\
\mathbf{T}_{\varphi \rho} & \mathbf{T}_{\varphi \varphi} & \mathbf{T}_{\varphi z} \\
\mathbf{T}_{z \rho} & \mathbf{T}_{z \varphi} & \mathbf{T}_{z z}
\end{array}\right]\left[\begin{array}{c}
0 \\
\mathbf{J}_{0 \varphi} \\
0
\end{array}\right]=\left[\begin{array}{c}
\mathbf{T}_{\rho \varphi} \\
\mathbf{T}_{\varphi \varphi} \\
\mathbf{T}_{z \varphi}
\end{array}\right] \mathbf{J}_{0 \varphi},
$$

where $\mathbf{T}=\mathbf{S}^{-1}$. Now, since the Green dyad is diagonally dominant, the system matrix $\mathbf{S}$ and its inverse $\mathbf{T}$ are diagonally dominant as well. But the two dominant terms $\mathbf{T}_{\rho \rho}$ and $\mathbf{T}_{z z}$ do not play any role, as $\mathbf{J}_{0 \rho}=\mathbf{J}_{0 z}=0$, while $\mathbf{T}_{\varphi \varphi}$ is dominant over $\mathbf{T}_{\rho \varphi}$ and $\mathbf{T}_{z \varphi}$, then $\mathbf{J}_{\varphi} \gg \mathbf{J}_{\rho}, \mathbf{J}_{z}$. Accordingly, one is able to approximate the current by its azimuthal component with expected fair accuracy. So, (6) is reduced to its single azimuthal component version:

$$
\begin{aligned}
& J_{\varphi}^{\mathrm{inc}}(\vec{r}) \simeq J_{\varphi}(\vec{r})- \\
& j \omega \mu_{0} \sigma_{0} \chi(\vec{r}) \int_{V_{f}} \overline{\overline{\mathbf{G}}}_{\varphi \varphi}^{(e e)}\left(\vec{r}, \vec{r}^{\prime}\right) J_{\varphi}\left(\vec{r}^{\prime}\right) d V^{\prime} .
\end{aligned}
$$

The variation of the mutual impedance (7) then becomes:

$$
Z \simeq-\frac{1}{I_{1} I_{2}} \int_{V_{f}} E_{\varphi, R x}^{\mathrm{inc}}\left(\vec{r}^{\prime}\right) J_{\varphi}\left(\vec{r}^{\prime}\right) d V^{\prime} .
$$

The error on the impedance due to this approximation is considered in section IV.

\section{ShAPE RECONSTRUCTION PROBLEM}

Let $\Omega$ be the prescribed RoI in the tube wall, and $V_{f}$, such as $V_{f} \subset \Omega$, be the damaged domain sought within it. One aims at the recovery of the shape boundary of the defect, $\partial V_{f}$. To achieve this task by a gradient-based approach, an initial shape is evolved into a direction that minimizes a functional misfit
TABLE I

FUNCTION SPACES REFERRED TO IN THE TEXT.

\begin{tabular}{ll}
\hline $\mathrm{C}$ & Space of contrast functions $\chi$ \\
$\mathrm{U}$ & Space of fictitious currents $\vec{J}$ \\
$\mathrm{Y}$ & Space of current sources $\vec{J}^{i n c}$ \\
$\mathrm{Z}$ & Space of measurements $\zeta$ \\
$\mathrm{S}$ & Space of level-set functions $\phi$ \\
\hline
\end{tabular}

of the 'measured' and the model data. An efficient approach enabling topological changes is to implicitly describe $V_{f}$ in terms of a level-set function. Adopting the notation used in [2], [19], the latter reads as

$$
\left\{\begin{array}{ll}
\phi(\vec{r}) \geq 0 & \text { for all } \quad \vec{r} \in V_{f} \\
\phi(\vec{r})<0 & \text { for all } \quad \vec{r} \in \Omega \backslash V_{f}
\end{array}\right\},
$$

such that the shape boundary is the zero level set:

$$
\partial V_{f}=\{\vec{r} \in \Omega, \phi(\vec{r})=0\} .
$$

\section{A. The operators}

The main operators used are defined below (let us refer to the function spaces in Table I).

Fictitious currents $\vec{J}_{i}(\vec{r})$ in $V_{f}$ due to current sources $\vec{J}_{i}^{i n c}(\vec{r})(6)$, where $i=1, \ldots, I$ correspond to the applied source currents, involve the operator $\Lambda(\chi): \mathrm{U} \mapsto \mathrm{Y}$, which, after discretization, corresponds to the linear system

$$
\Lambda(\chi) \vec{J}_{i}=\vec{J}_{i}^{i n c} .
$$

Measurement operators $\mathcal{M}_{i j}: \mathrm{U} \mapsto \mathrm{Z}$, where $\mathrm{Z}=\mathbb{C}$, map the current $\vec{J}_{i}$ for the transmitter $\mathrm{Tx}_{i}$ onto a measurement $Z_{i j}$ for the receiver $\mathrm{Rx}_{j}$ (8),

$$
Z_{i j}=\mathcal{M}_{i j} \overrightarrow{J_{i}}
$$

Accordingly, the measured 'experimental' data $\zeta_{i j} \in \mathrm{Z}$ are associated to the current $\vec{J}_{i}^{\text {true }}$ corresponding to the 'true' defect $\chi^{\text {true }}(\vec{r})$,

$$
\zeta_{i j}=\mathcal{M}_{i j} \vec{J}_{i}^{\text {true }} .
$$

The forward operator $\mathcal{A}_{i j}: \mathrm{C} \mapsto \mathrm{Z}$ maps contrast functions $\chi$ onto the data space,

$$
\mathcal{A}_{i j}(\chi)=\mathcal{M}_{i j} \Lambda(\chi)^{-1} \vec{J}_{i}^{i n c} .
$$

The residual operator $\mathcal{R}_{i j}: \mathrm{C} \mapsto \mathrm{Z}$ is

$$
\mathcal{R}_{i j}(\chi)=\mathcal{A}_{i j}(\chi)-\zeta_{i j} .
$$

Defining a nonlinear operator $\Pi: \mathrm{S} \mapsto \mathrm{C}$ that maps the levelset function onto the contrast function

$$
\chi(\vec{r})=\Pi(\phi)(\vec{r})=\left\{\begin{array}{ll}
-1, & \phi(\vec{r}) \geq 0 \\
0, & \phi(\vec{r})<0
\end{array}\right\},
$$

the residual operator (19) is written as

$$
\mathcal{T}_{i j}(\phi)=\mathcal{R}_{i j}(\Pi(\phi)) .
$$

The shape reconstruction problem is then formulated as the retrieval of the level-set function $\phi$ which minimizes a leastsquare functional $\mathcal{F}(\phi)$

$$
\mathcal{F}(\phi)=\sum_{i=1}^{I} \sum_{j=1}^{L} \mathcal{F}_{i j}(\phi)=\sum_{i=1}^{I} \sum_{j=1}^{L} \frac{1}{2} \overline{\mathcal{T}_{i j}(\phi)} \mathcal{T}_{i j}(\phi),
$$

where overbar means complex conjugate. 


\section{B. Differential operators}

Solution of (22) via a gradient-based approach requires the calculation of the gradient of the cost function with respect to the level-set function. An analytical derivation of the gradient with respect to the contrast function has been proposed in [11] using function space methods. One then defines the gradient in harmony with this result and deals with the level-set function only at the last stage.

For the calculation of the gradient, the concept of Gateaux derivative as a generalization of the discrete directional derivative is employed. The derivative of $\mathcal{F}_{i j}$ along the direction given by an incremental $\delta \phi$ is expressed as the dot product between the gradient $\operatorname{grad}_{\phi} \mathcal{F}_{i j}(\phi): \mathrm{S} \mapsto \mathbb{R}$ and the increment:

$$
d_{\delta \phi} \mathcal{F}_{i j}(\phi)=\left.\frac{d}{d \beta} \mathcal{F}_{i j}(\phi+\beta \delta \phi)\right|_{\beta=0}=\operatorname{grad}_{\phi} \mathcal{F}_{i j}(\phi) \cdot \delta \phi,
$$

It can be shown that (23) transforms into

$$
d_{\delta \phi} \mathcal{F}_{i j}(\phi)=\Re\left(\overline{\mathcal{T}_{i j}(\phi)} \operatorname{grad}_{\phi} \mathcal{A}_{i j}(\Pi(\phi)) \delta \phi\right),
$$

$\Re$ as the real part.

The calculation of $\operatorname{grad}_{\phi} \mathcal{A}_{i j}(\Pi(\phi))$ follows from the one of the gradient of the forward operator with respect to the contrast function, $\operatorname{grad}_{\chi} \mathcal{A}_{i j}(\chi)$, which is proposed in general form in [11].

Similarly to (23), $\operatorname{grad}_{\chi} \mathcal{A}_{i j}(\chi)$ is introduced as a linear operator that maps a change of contrast function onto a change of impedance, $\operatorname{grad}_{\chi} \mathcal{A}_{i j}(\chi): \mathrm{C} \mapsto \mathrm{Z}$,

$$
d_{\delta \chi} \mathcal{A}_{i j}(\chi)=\left.\frac{d}{d \beta} \mathcal{A}_{i j}(\chi+\beta \delta \chi)\right|_{\beta=0}=\operatorname{grad}_{\chi} \mathcal{A}_{i j}(\chi) \cdot \delta \chi .
$$

Proceeding as in [11], for the multi-static configuration of our interest (driving and receiver coils are different), one differentiates the variation of impedance $Z_{i j}$ in (8) as

$$
d_{\delta \chi} Z_{i j}(\chi)=-\frac{1}{I_{1} I_{2}} \int_{V_{f}} \vec{E}_{R x_{j}}^{i n c}\left(\vec{r}^{\prime}\right) \cdot d_{\delta \chi} \vec{J}_{i}\left(\vec{r}^{\prime}, \chi\right) d V^{\prime}
$$

Since $\vec{E}_{R x_{j}}^{i n c}$ is the primary field associated to the receiver $\vec{E}_{R x_{j}}^{i n c}$, one introduces an adjoint field $\vec{E}_{R x_{j}}$ as the electric field obtained by interchanging indices $\vec{r}$ and $\vec{r}^{\prime}$ and solving (2) for $\vec{E}_{R x_{j}}^{i n c}$, i.e.,

$$
\begin{aligned}
& \vec{E}_{R x_{j}}^{i n c}\left(\vec{r}^{\prime}\right)=\vec{E}_{R x_{j}}\left(\vec{r}^{\prime}\right)- \\
& j \omega \mu_{0} \int_{V_{f}} d V\left[\overline{\overline{\mathbf{G}}}^{(e e)}\left(\vec{r}, \vec{r}^{\prime}\right)\right]^{T} \cdot \vec{J}_{R x_{j}}(\vec{r}) .
\end{aligned}
$$

Differentiating $\vec{J}$ as a function of the contrast yields

$$
\begin{aligned}
& d_{\delta \chi} \vec{J}^{i n c}\left(\vec{r}^{\prime}, \chi\right)=\sigma \vec{E}(\vec{r}) \delta \chi(\vec{r})+ \\
& j \omega \mu_{0} \sigma_{0} \chi(\vec{r}) \int_{V_{f}} d V^{\prime} \overline{\overline{\mathbf{G}}}^{(e e)}\left(\vec{r}, \vec{r}^{\prime}\right) \cdot d_{\delta \chi} \vec{J}\left(\vec{r}^{\prime}, \chi\right) .
\end{aligned}
$$

Then, substituting (27) into (26), reordering terms, using the dyadic relationship (12), and identifying the differential (28), one gets

$$
\begin{aligned}
& \left(I_{1} I_{2}\right) d_{\delta \chi} Z_{i j}(\chi)=-\int_{V_{f}} d V \vec{E}_{R x_{j}}(\vec{r}) \cdot d_{\delta \chi} \vec{J}_{i}(\vec{r}, \chi)+ \\
& \int_{V_{f}} d V \vec{E}_{R x_{j}}(\vec{r}) j \omega \mu_{0} \sigma_{0} \chi(\vec{r}) \int_{V_{f}} d V^{\prime} \overline{\overline{\mathbf{G}}}^{(e e)}\left(\vec{r}, \vec{r}^{\prime}\right) d_{\delta \chi} \vec{J}_{i}\left(\vec{r}^{\prime}, \chi\right) \\
& =-\int_{V_{f}} d V \vec{E}_{R x_{j}}(\vec{r}) \cdot \vec{E}_{i}(\vec{r}) \sigma_{0} \delta \chi(\vec{r}), \\
& \text { i.e., } \\
& \qquad d_{\delta \chi} \mathcal{A}_{i j}(\chi)=-\frac{\sigma_{0}}{I_{1} I_{2}} \int_{V_{f}} \vec{E}_{R x_{j}}\left(\vec{r}^{\prime}\right) \cdot \vec{E}_{i}\left(\vec{r}^{\prime}\right) d V^{\prime} \delta \chi
\end{aligned}
$$

and the gradient $\operatorname{grad}_{\chi} \mathcal{A}_{i j}(\chi)$ is

$$
\operatorname{grad}_{\chi} \mathcal{A}_{i j}(\chi)=-\frac{\sigma_{0}}{I_{1} I_{2}} \int_{V_{f}} \vec{E}_{R x_{j}}\left(\vec{r}^{\prime}\right) \cdot \vec{E}_{i}\left(\vec{r}^{\prime}\right) d V^{\prime} .
$$

The level-set function is now introduced from the relationship between the contrast (20) and a one-dimensional Heaviside function, $\chi=\Pi(\phi)=-H(\phi)$, such that the DiracDelta function is $\delta(\phi)=\partial H(\phi) / \partial \phi$, then

$$
d \chi=-\delta(\phi) \delta \phi
$$

where an infinitesimal variation of the contrast function is understood from a variation of the level-set function, $\delta \chi=$ $H(\phi+\delta \phi)-H(\phi)$. Making explicit the dependency $\chi=\chi(\phi)$ in (30) and using (32),

$$
d_{\delta \phi} \mathcal{A}_{i j}(\phi)=\frac{\sigma_{0}}{I_{1} I_{2}} \int_{V_{f}} \vec{E}_{R x_{j}}\left(\vec{r}^{\prime}\right) \cdot \vec{E}_{i}\left(\vec{r}^{\prime}\right) d V^{\prime} \delta(\phi) \delta \phi .
$$

The gradient of the cost function then reads as

$$
\begin{aligned}
& \operatorname{grad} \mathcal{F}_{i j}(\phi)=\frac{\sigma_{0}}{I_{1} I_{2}} \\
& \Re\left(\overline{\mathcal{T}_{i j}(\phi)} \int_{V_{f}} \vec{E}_{R x_{j}}\left(\vec{r}^{\prime}\right) \cdot \vec{E}_{i}\left(\vec{r}^{\prime}\right) d V^{\prime} \delta(\phi)\right) .
\end{aligned}
$$

The Dirac delta $\delta(\phi)$ in (33) means integration on the defect boundary $\partial V_{f}$. Thus, the $\operatorname{gradient}, \operatorname{grad}_{\phi} \mathcal{A}_{i j}(\phi)$, resulting from (33) is not valued on the whole domain, which is an obvious complication for a gradient-based method [8], [19]. An alternative involves the approximation of the Dirac-delta in (32) by a function $\Xi$, set to 1 in a small finite-width neighborhood of $\partial V_{f}$ and to 0 elsewhere, up to some constants. Then,

$$
\operatorname{grad}_{\phi} \mathcal{A}_{i j}(\phi) \propto \int_{V_{f}} \vec{E}_{R x_{j}}\left(\vec{r}^{\prime}\right) \cdot \vec{E}_{i}\left(\vec{r}^{\prime}\right) d V^{\prime} \Xi\left(\partial V_{f}\right) .
$$

In the numerical implementation herein, even though $\Xi\left(\partial V_{f}\right)$ in (35) is zero far from the defect boundary, the numerical computation of $\operatorname{grad}_{\phi} \mathcal{A}_{i j}(\phi)$ is extended into the whole domain, i.e., $\Xi=1$ everywhere, avoiding the determination of a specific neighborhood of $\partial V_{f}$. (Let us notice here that this amounts to the extension of the velocity onto the whole domain in a Hamiltonian approach of a level-set evolution [7].) 


\section{Implementation of the algorithm}

The most important features of the implementation of the proposed solution method are described below.

One starts from a level-set function as a piecewise constant function the value of which at cell $n$ is $\phi_{n}$, with $n=1, \ldots, N$. The directional derivative is accordingly given by

$$
d_{\delta \phi} \mathcal{F}_{i j}(\phi)=\sum_{n=1}^{N} \operatorname{grad}_{\phi_{n}} \mathcal{F}_{i j}(\phi) \delta \phi_{n}
$$

such that $\operatorname{grad}_{\phi_{n}} \mathcal{F}_{i j}(\phi)=\partial \mathcal{F}_{i j}(\phi) / \partial \phi_{n}$. Now, let us consider this discrete level-set representation at some iteration $k$, $\phi\left(\vec{r}_{n}\right)^{[k]}$, the initial guess $(k=0)$ being chosen as a signed distance function from each point $\vec{r}_{n}$ to the surface of a sphere with center at initial location $\vec{r}_{0}$ and radius $R_{0}$,

$$
\phi\left(\vec{r}_{n}\right)^{[0]}=R_{0}^{2}-\left|\vec{r}_{n}-\vec{r}_{0}\right|^{2} .
$$

The update of the level set-function at step $k+1$ is given by

$$
\phi_{n}^{[k+1]}=\phi_{n}^{[k]}+\alpha^{[k+1]} p_{n}^{[k]},
$$

where $\alpha$ is a chosen step size and $p_{n}$ is the search direction set as the Polak-Ribiere conjugate gradient direction

$$
p_{n}^{[k+1]}=-\nabla \mathcal{F}_{n}^{[k+1]}+\beta^{[k+1]} p_{n}^{[k]},
$$

where

$$
\beta^{[k+1]}=\max \left\{\frac{\sum_{n=1}^{N}\left(\nabla \mathcal{F}_{n}^{[k+1]}-\nabla \mathcal{F}_{n}^{[k]}\right) \nabla \mathcal{F}_{n}^{[k+1]}}{\sum_{n=1}^{N} \nabla \mathcal{F}_{n}^{[k]} \nabla \mathcal{F}_{n}^{[k]}}, 0\right\},
$$

and $p_{n}^{[0]}=-\nabla \mathcal{F}_{n}^{[0]}$.

The step size $\alpha$ should be such that the defect domain (the contrast) is changed by between one and three voxels at each update. This certainly slows down the reconstruction yet yields a stable evolution of the level-set function [2], [20] - if the constraint cannot be satisfied, the upper bound is relaxed, enabling more than three voxels to change.

In addition, the inverse solution is regularized by smoothing the search direction $p$ via an uniform spatial filter, previously to its update (38). In practice, its output is the average of its 26 neighbors, 6 sharing a face, 12 an edge, and 8 a node, this procedure being applied recursively three times — smoothing yields as a general observation a more compactly retrieved defect, and all results thereafter incorporate it.

The above procedure is pursued until the cost functional (22) cannot be reduced any further, as is done in [2].

\section{Comparison with a binary-specialized, non-linear conjugate-gradient method}

Results obtained by the proposed level-set retrieval are compared in section IV with those provided by a binaryspecialized, non-linear conjugate-gradient method (denoted as BNLCG). Since the derivative of the cost function with respect to a binary contrast function is not defined, a relaxed version of the contrast function which goes continuously from 0 to 1 can be put together (refer to [21] for its initial setting in a
2-D scattering case, and [12] for its development for a 3-D eddy-current case). The contrast function $\chi(\vec{r})$ reads as

$$
\chi(\vec{r})=\frac{\sigma^{*}-\sigma_{0}}{\sigma_{0}} \Psi(\tau(\vec{r})), \quad \Psi(\tau(\vec{r}))=\frac{1}{1+\mathrm{e}^{-\frac{\tau(\vec{r})}{\theta}}},
$$

where $\sigma^{*}$ is known (in the present case, this is air with $\sigma^{*}=$ $0)$. The function $\Psi(\tau(\vec{r}))$ varies from 0 to 1 when $\tau(\vec{r})$ varies from $-\infty$ to $+\infty$ (here $\theta$ is kept constant and equated to one). Then, one aims at the minimization of a cost function $\tilde{\mathcal{F}}(\tau)$ with respect to $\tau$ (variation with $\vec{r}$ is implied) stated as

$$
\tilde{\mathcal{F}}(\tau)=\sum_{i=1}^{I} \sum_{j=1}^{L} \tilde{\mathcal{F}}_{i j}(\tau)=\sum_{i=1}^{I} \sum_{j=1}^{L} \frac{1}{2} \overline{\mathcal{R}_{i j}(\tau)} \mathcal{R}_{i j}(\tau) .
$$

The gradient of $\tilde{\mathcal{F}}_{i j}$ in (42), like in (24), is valued to

$$
\operatorname{grad}_{\tau} \tilde{\mathcal{F}}_{i j}(\tau)=\Re\left(\chi^{\prime}(\tau) \overline{\mathcal{R}_{i j}(\tau)} \operatorname{grad}_{\chi} \mathcal{A}_{i j}(\chi)\right),
$$

where $\chi^{\prime}(\tau)$ is the derivative of $\chi$ with respect to $\tau$ and $\operatorname{grad}_{\chi} \mathcal{A}_{i j}(\chi)$ is given by (31). Update of the contrast function is made according to a Polak-Ribiere conjugate-gradient scheme, as in (39) upon substitution of $\tilde{\mathcal{F}}$ to $\mathcal{F}$, the step size being computed analytically as suggested in [11].

\section{NUMERICAL RESULTS}

The algorithm has been thoroughly tested from synthetic data, a small set of illustrative results being analyzed herein. The configuration itself is taken from [13]. The tube wall is a highly conductive, non-magnetic material (Inconel 600: conductivity $\sigma_{0}=0.98 \times 10^{6} \mathrm{Sm}^{-1}$ ), and it is of internal and external radii of 9.83 and $11.1 \mathrm{~mm}$, resp. The region of interest inside has dimensions $\Delta \rho \times \Delta \varphi \times \Delta z=1.27 \mathrm{~mm} \times 8^{\circ} \times 1.6 \mathrm{~mm}$ and is divided into $10 \times 16 \times 16=2560$ cells, each of size $\Delta \rho \times \Delta \varphi \times \Delta z=0.127 \mathrm{~mm} \times 0.5^{\circ} \times 0.1 \mathrm{~mm}$. The source is a 30-turn circular coil of internal and external radii of 9.25 and $9.55 \mathrm{~mm}, 2 \mathrm{~mm}$ thick, centered along the tube axis. The variation of impedance between the source coil and a small coil receiver (assumed as a vertical dipole) is measured for 16 heights of the source coil and, for each source position, 16 positions of the receiver coil along a small portion of a circular arc at $9.73 \mathrm{~mm}$ from the center of the tube, scanning a total surface of $\Delta \varphi \times \Delta z=8^{\circ} \times 1.6 \mathrm{~mm}$ with step $\delta \varphi \times \delta z=0.5^{\circ} \times 0.1 \mathrm{~mm}$. Two frequencies are employed, $100 \mathrm{kHz}$ (skin depth $\delta=1.6 \mathrm{~mm})$ and $500 \mathrm{kHz}(\delta=0.72 \mathrm{~mm})$.

The case of a void defect opening in the interior of the tube (inner defect) is illustrated in figure 1.

It can be a shallow defect (dimensions of $\Delta \rho \times \Delta \varphi \times \Delta z=$ $0.51 \mathrm{~mm} \times 3.5^{\circ} \times 0.6 \mathrm{~mm}$, its depth as $40 \%$ thickness of the wall, Fig. 1(a)) or a deep defect (dimensions of $\Delta \rho \times \Delta \varphi \times \Delta z=$ $1 \mathrm{~mm} \times 0.65 \mathrm{~mm} \times 0.65 \mathrm{~mm}$, its depth as $83 \%$ thickness of the wall, Fig. 1(e)), several reconstructions being proposed, further results (the decrease of the cost function and its gradient) being found in Figs. 3(a)-3(b)). Discretization into $10 \times 16 \times 16$ cells and $10 \times 6 \times 6$ cells is respectively performed.

Letting the initial estimate be made of a few voxels in the center of the tube wall, and using data calculated with the same discretization as the one used along the inversion, the inner void defect appears well retrieved according both depth 


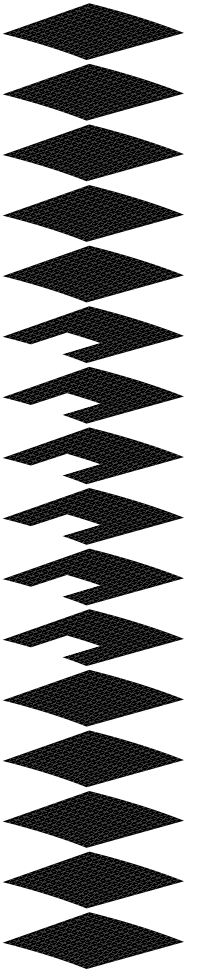

(a) Shallow void

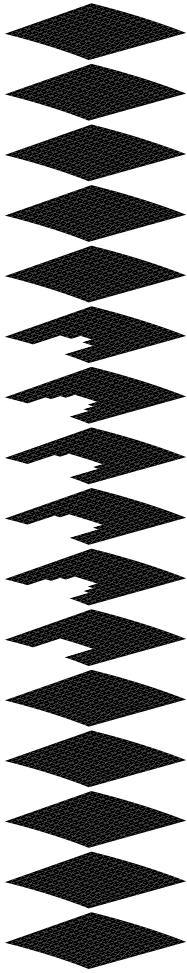

(b)

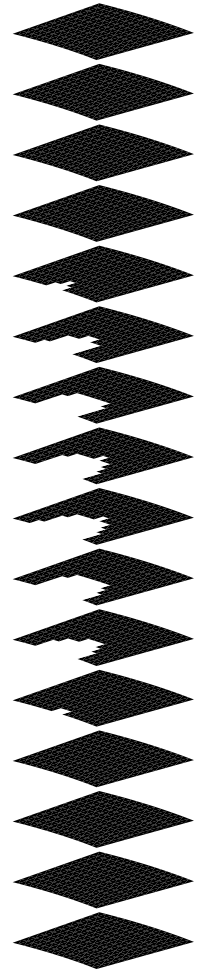

(c)

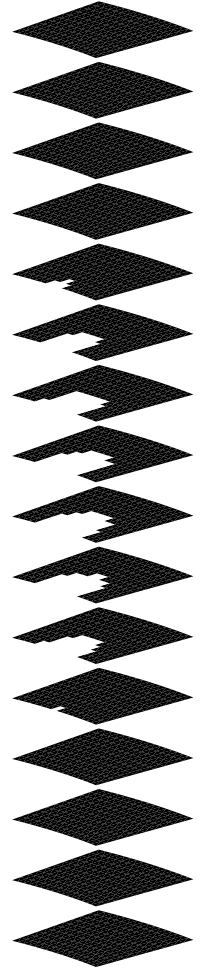

(d)

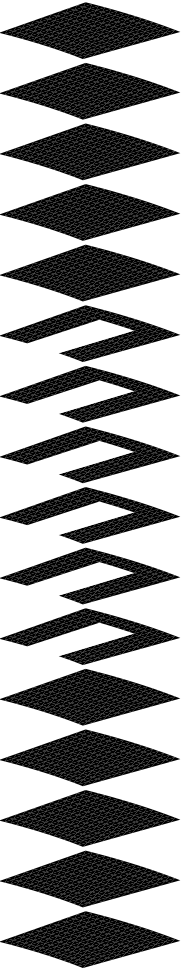

(e) Deep void

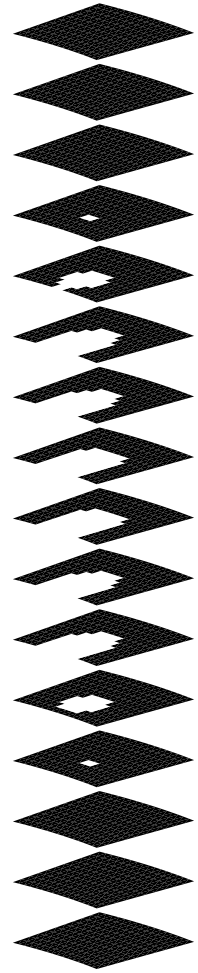

(f)

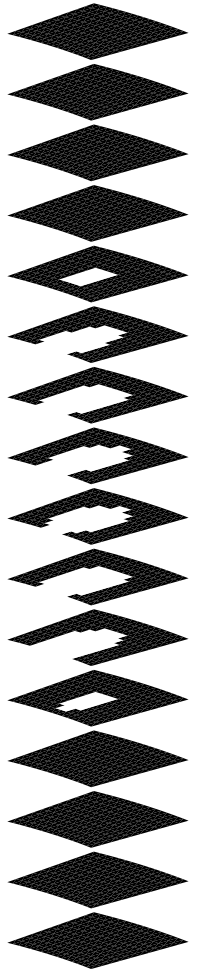

(g)

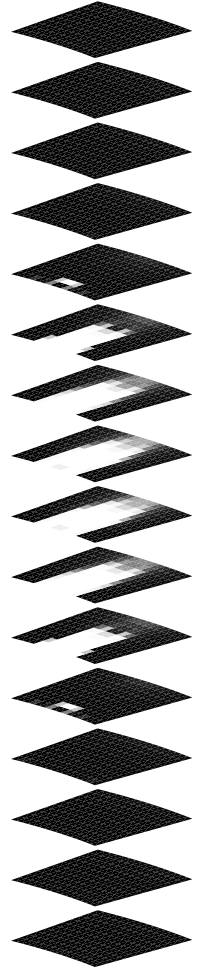

(h)

Fig. 1. Single inner void retrieved by the level-set method (LS) at $100 \mathrm{kHz}$, with comparison to a binary-specialized scheme (BNLCG). Slices of contrast $\chi$ along $z$ are displayed in the RoI (spanning the whole thickness of the wall), blank cells being as $\chi=1$ (defect) and black ones as $\chi=0$. Shallow defect: exact one (a), LS retrieved (b-d) from initial estimate in the center of the wall, with exact data (b), with noisy data and azimuthal approximation (c), and with noisy data without azimuthal approximation (d). Deep defect: exact one (e), LS retrieved (f-g) from initial estimate in the center of the wall and exact data, with azimuthal approximation (f) or without azimuthal approximation (g), and BNLCG retrieved (h).

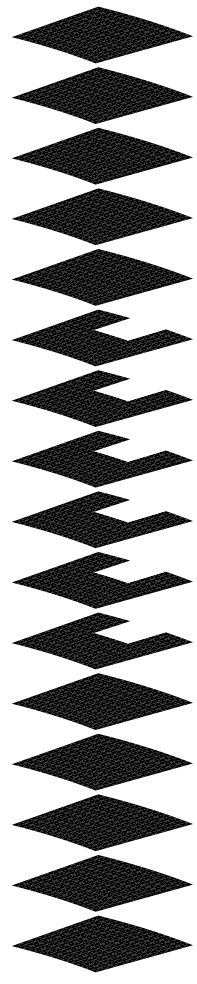

(a) Outer void

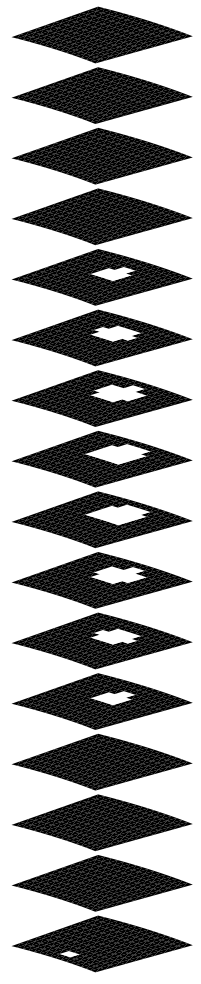

(b)

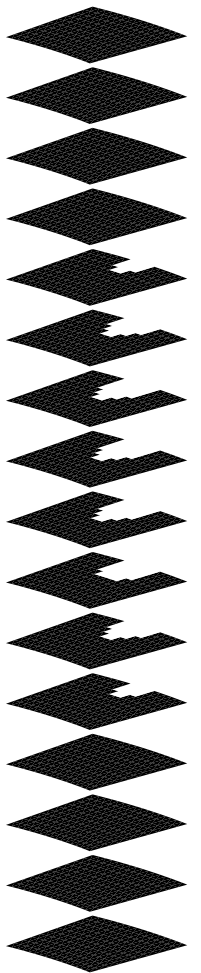

(c)

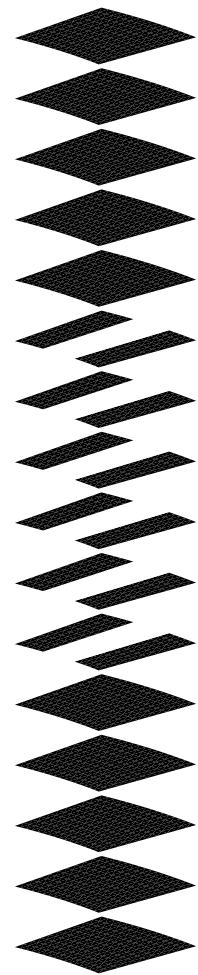

(d) Through-wall

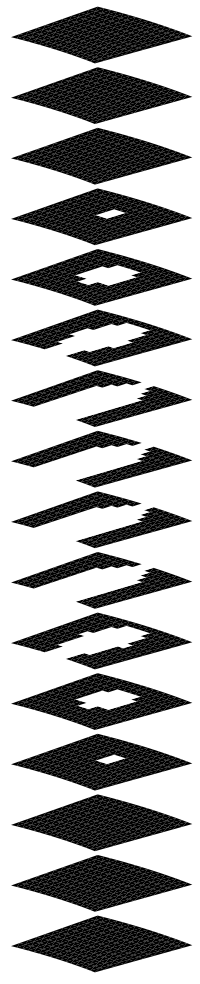

(e)

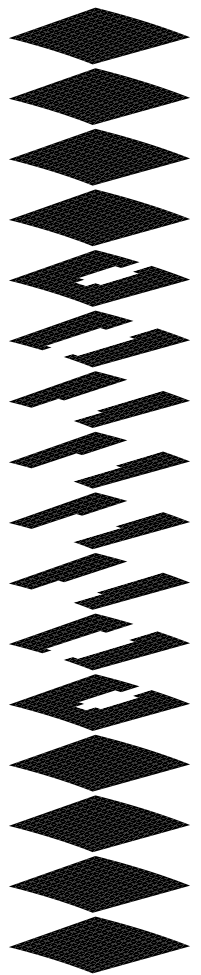

(f)

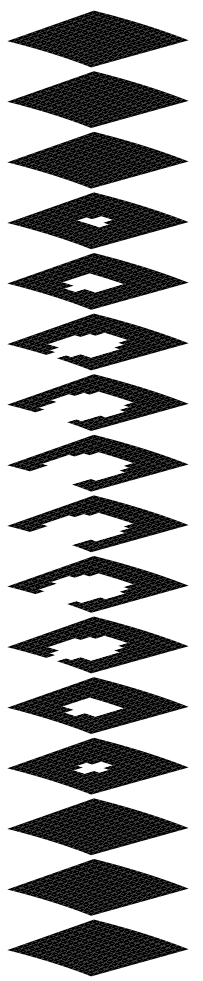

(g)

Fig. 2. Single outer or through-wall void defect retrieved by the level-set method (LS) (same display as in Fig. 1): exact outer defect (a), retrieved from initial estimate in the center of the wall (b) or at its exterior interface (c); exact through-wall defect (d), retrieved from initial estimate in the center of the wall (e), as a long inner defect (f), and at $500 \mathrm{kHz}$ in the center of the wall (g), $100 \mathrm{kHz}$ being assumed otherwise. 
and height, its azimuthal extent however being slightly overestimated. As for the cost function, it is decreased by three orders of magnitude after it reaches a plateau, as well as its gradient (Figs. 3(a)-3(b)).

Then, if Gaussian noise is added to both real and imaginary parts of the variations of impedance (11 dB SNR), the retrieved defect becomes rather blurred (Fig. 1(c)), in agreement with the fact that the cost function is only reduced by a factor of 10 from its initial value (its gradient is decreased down to the same level as without noise, no further improvement appears possible).

Let us notice in addition that the over-estimation of the azimuthal extent mentioned in the above appears at least partially caused by the azimuthal approximation made, since a slight improvement is gained when using the full field (6),(7); in particular one can compare Fig. 1(d) where this approximation is not made to Fig. 1(c) where it is.

Retrieval of a bigger defect is shown in figure 1(f)) with an error of about $0.25 \mathrm{~mm}(\delta / 6)$. Let us notice that the error on the variation of impedance due to the azimuthal approximation, taken as $100 \frac{\left\|d Z-d Z\left(J_{\varphi}\right)\right\|}{\|d Z\|} \mid$, is of the order of $3 \%$. Here, carrying out the reconstruction with the full fields instead of the approximated ones again slightly reduces over-shoot in the azimuthal direction.

The level-set method is also compared with the BNLCGmethod as summarized in the above. The fully binary representation of the defect by the level-set method can indeed be put in contrast with the continuous representation of the contrast by the BNLCG-method, see Fig. 1(h). Both in effect yield similar retrievals, save the fact that the minimum of the cost function reached by the latter method is two orders of magnitude lower than with the former (results not shown).

The case of a void defect identical with the one in the above but now opening in the exterior of the tube (outer defect) is illustrated in figure 2 as well as the case of a through-wall defect (dimensions $\Delta \rho \times \Delta \varphi \times \Delta z=1.27 \mathrm{~mm} \times 3.2^{\circ} \times 0.65 \mathrm{~mm}$, discretized into $16 \times 7 \times 7$, Fig. $2(d))$.

Retrievals of the outer defect, refer to Fig.2(b) from an initial estimate in the center of the tube, and to Fig. 2(c)) from an initial estimate at the exterior boundary, appear quite similar. As for the through-wall defect, full depth recovery is achieved as illustrated in Fig. 2(e). This is improved by taking a long defect as the initial estimate, the location of which could be assumed as a-priori information from the previous retrieval, refer to Fig. 2(f). Here, let us emphasize that increasing the frequency to $500 \mathrm{kHz}(\delta=0.72)$ instead of $100 \mathrm{kHz}$ in all above cases worsens the depth estimate as exemplified in Fig. $2(\mathrm{~g})$.

Considering now two defects, an inner one and an outer one, each of same size $\Delta \rho \times \Delta \varphi \times \Delta z=0.39 \mathrm{~mm} \times 3.2^{\circ} \times 0.65 \mathrm{~mm}$ and discretized into $5 \times 7 \times 7$ cells (Fig. 4(a)), only the inner defect could be retrieved (Fig. 4(b)) unless an initial estimate comprising two defect zones is chosen (Fig. 4(c)). In any case, the size of the outer defect is over-estimated and the size of the inner one is under-estimated in relation to the fact that the sensitivity of the variation of impedance with respect to the contrast decreases with depth.

With the BNLCG method, the retrieval of two defects is feasible, without prior information on the location of the defect (results not shown). This can be a mixed effect between a low sensitivity to changes near the exterior interface of the tube and the propagative nature of the level-set method from the initial estimate. Further insight into the sensitivity of the variation of impedance with respect to the level-set function, i.e., the Jacobian matrix, confirms the large decrease in sensitivity faced with depth as shown in Fig. 5(a) for a typical measurement set-up (one of those used to get the results of Fig. 1).

\section{Discussion}

The feasibility of the retrieval of 3-D void defects is investigated by means of a gradient-based, level-set method. Both inner and outer defects, as well as a through-hole one are fairly mapped. Yet two defects opening in air, one inner one and one outer one; are retrieved only when two defects are also assumed as the initial estimate. As a general rule, a decrease of sensitivity with depth, and so a better mapping of inner defects is observed. Reduction of dimensionality of the inversion by approximating the fictitious current to its azimuthal component leads to a few-percent error on the variation of impedance, and is successfully for most retrievals - the error is the largest for long defects, yielding slightly over-extended ones along the azimuthal direction.

Reconstruction with the BNLCG method, a detailed investigation of which is out of the scope of this contribution, leads to rather similar results, yet (in general) the minimum of the cost function reached is much smaller. This may be due to the fact that the BNLCG method updates the contrast in the whole region of interest at every iteration whereas the proposed level-set method evolves the shape from an initial guess and suffer from occurence of regions of less sensitivity to contrast changes.

Dimensionality and computation time are major issues due to the size of the Green dyads involved; for a discretization of the search domain into $10 \times 16 \times 16$ cells, these dyads require 1 GB of memory. Yet, since the contrast function is zero outside the defect, inversion of the linear system and matrix-vector multiplication involving the contrast can be speeded up by restriction to the defect domain.

Further improvement of the algorithm could result from a two-step approach for which an estimate of the defect location is obtained by means of a fast volume reconstruction method, whilst normalizing the gradients should improve sensitivity with depth, e.g., [19].

\section{ACKNOWLEDGMENT}

The eddy-current part of the CIVA platform has been employed, courtesy of CEA LIST, Saclay. The first author has been supported by a one-year grant (2007-2008) from Région Île-de-France.

\section{REFERENCES}

[1] A. Skarlatos, G. Pichenot, D. Lesselier, M. Lambert, and B. Duchêne, "Modeling of eddy-current interactions with flaws in ferromagnetic tubes via an integral equation formalism," IEEE Trans. Magn., vol. to appear, 2008. 


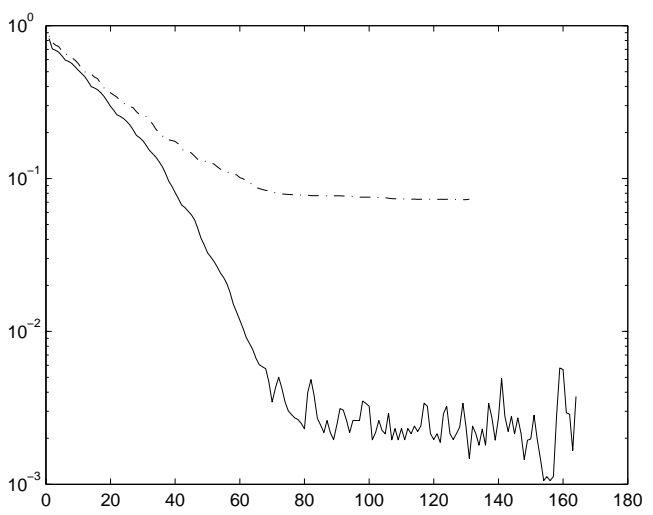

(a) Cost function

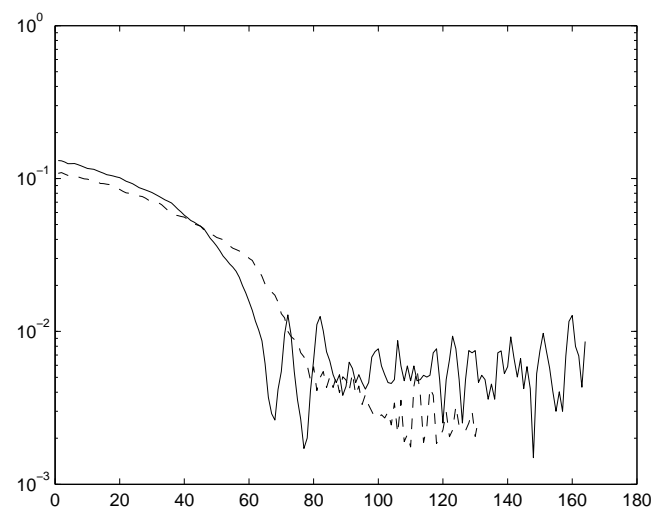

(b) Gradient of the cost function

Fig. 3. Evolution of the cost function and its gradient as a function of the number of iterations when carrying out the retrieval of an inner defect from exact data (full line) and noisy ones (11dB SNR) (dash line), with reference to Fig.1.

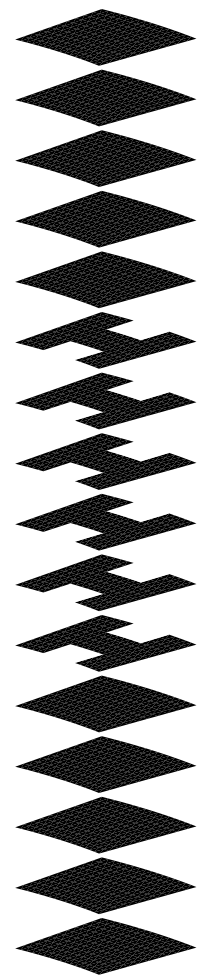

(a) Two defects

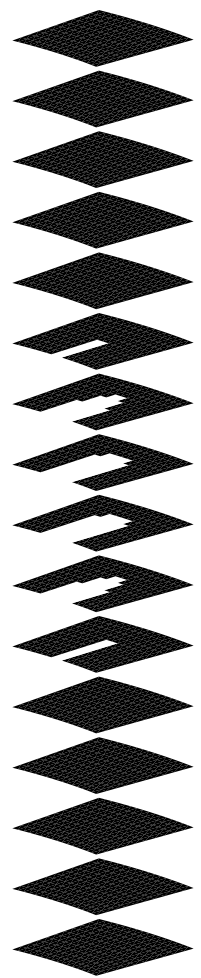

(b)

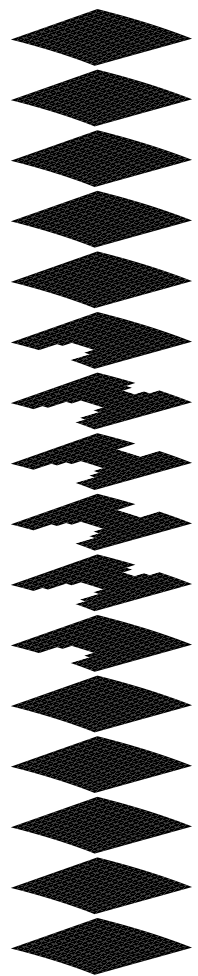

(c)
Fig. 4. Two defects, an inner one and an outer one, retrieved by the level-set method (LS): exact ones (a), retrieved from initial estimate in the center of the wall (b) or from initial estimate made of distinct zones, an inner one and an outer one (c).

[2] O. Dorn and D. Lesselier, "Level set methods for inverse scattering," Inv. Problems, vol. 22, pp. R67-R131, 2006.

[3] J. A. Sethian, Level Set Methods and Fast Marching Methods. Cambridge, UK: Cambridge University Press, 1999.

[4] S. Osher and R. Fedkiw, Level Set Methods and Dynamic Implicit Surfaces. New York: Springer-Verlag, 2003.

[5] F. Santosa, "A level set approach for inverse problems involving obstacles," ESAIM Control Optim. Calculus Variations, vol. 1, pp. 17-33, 1996.

[6] J. Sokolowski and J. P. Zolésio, Introduction to Shape Optimization. Shape Sensitivity Analysis. New York: Springer-Verlag, 1992.

[7] C. Ramananjaona, M. Lambert, D. Lesselier, and J.-P. Zolésio, "Shape

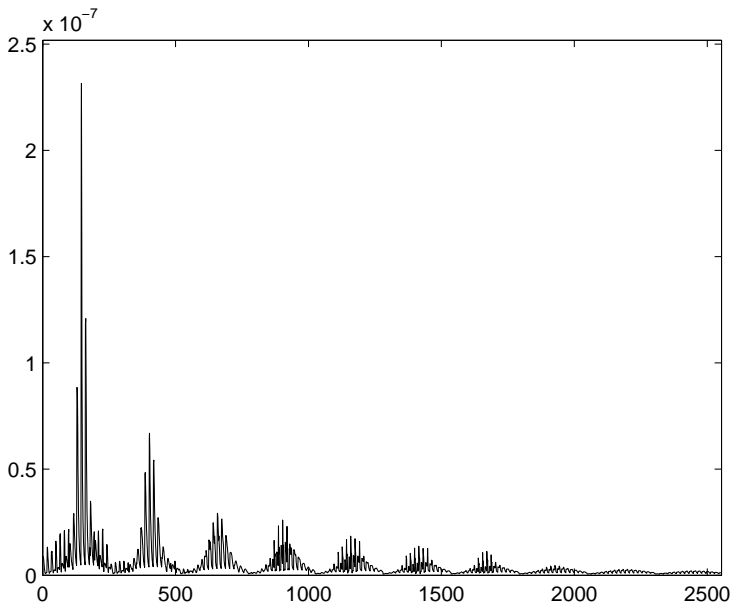

Fig. 5. Magnitude of the Jacobian at cell centers (numbered from left to right in the azimuthal direction, at increasing depth) for a typical measurement set-up.

reconstruction by controlled evolution of a level set: from a min-max formulation to numerical experimentation," Inverse Problems, vol. 17, pp. 1087-1111, 2001

[8] O. Dorn, E. L. Miller, and C. M. Rappaport, "A shape reconstruction method for electromagnetic tomography using adjoint fields and level sets," Inv. Problems, vol. 16, pp. 1119-1156, 2000.

[9] K. van den Doel and U. M. Ascher, "Dynamic level set regularization for large distributed parameter estimation problems," Inverse Problems, vol. 23, pp. 1271-1288, 2007.

[10] M. Burger, "Levenberg-marquardt level set methods for inverse obstacle problems," Inv. Problems, vol. 20, pp. 259-282, 2004

[11] S. J. Norton and J. R. Bowler, "Theory of eddy current inversion," $J$. Appl. Phys., vol. 73, pp. 501-512, 1993.

[12] D. Dos Reis, M. Lambert, and D. Lesselier, "Eddy-current evaluation of three-dimensional defects in a metal plate," Inverse Problems, vol. 18, pp. 1857-1871, 2002

[13] V. Monebhurrun, B. Duchêne, and D. Lesselier, "Three-dimensional inversion of eddy current data for non-destructive evaluation of steam generator tubes," Inv. Problems, vol. 14, pp. 707-724, 1998.

[14] W. C. Chew, Waves and Fields in Inhomogeneous Media. New York: IEEE Press, 1995.

[15] A. T. de Hoop, Handbook of Radiation and Scattering of Waves. Delft: Academic Press, 1995

[16] A. Abubakar and P. M. van den Berg, "Iterative forward and inverse 
algorithms based on domain integral equations for three-dimensional electric and magnetic objects," J. Comp. Phys., vol. 195, pp. 236-262, 2004.

[17] C. V. Dodd and W. E. Deeds, "Integral solutions to some eddy-current problems," Int. J. Nondestruct. Test., vol. 1, pp. 29-90, 1969.

[18] B. A. Auld, "Theoretical characterization and comparison of resonantprobe microwave eddy-current testing with conventional low-frequency eddy-current methods," in Eddy-Current Characterization of Material and Structures, G. Birnbaum and G. Free, Eds., American Society for Testing and Materials, Ed., vol. 12, 1981, pp. 332-347.

[19] O. Dorn, "Shape reconstruction in scattering media with voids using a transport model and level sets," Canad. Appl. Math. Quart., vol. 10, pp. 239-275, 2002.

[20] — "Scattering and absorption transport sensitivity functions for optical tomography," Optics Express, vol. 7, pp. 492-506, 2000.

[21] L. Souriau, B. Duchêne, D. Lesselier, and R. E. Kleinman, "Modified gradient approach to inverse scattering for binary objects in stratified media," Inverse Problems, vol. 12, pp. 463-481, 1996. 